FTUAM 01/07

IFT-UAM/CSIC-01-13

HIP-2001-08/TH

SUSX-TH/01-017

April 2001

\title{
Muon anomalous magnetic moment in supersymmetric scenarios with an intermediate scale and nonuniversality
}

\author{
D.G. Cerdeño ${ }^{1,2}$, E. Gabrielli ${ }^{3}$, S. KhaliL ${ }^{4,5}$, C. Muñoz ${ }^{1,2}$, E. Torrente-Lujan ${ }^{1}$ \\ 1 Departamento de Física Teórica C-XI, Universidad Autónoma de Madrid, \\ Cantoblanco, 28049 Madrid, Spain. \\ ${ }^{2}$ Instituto de Física Teórica C-XVI, Universidad Autónoma de Madrid, \\ Cantoblanco, 28049 Madrid, Spain. \\ ${ }^{3}$ Helsinki Institute of Physics, P.O. Box 64, FIN-00014 Helsinki, Finland. \\ ${ }^{4}$ Centre for Theoretical Physics, University of Sussex, Brighton BN1 9QJ, U.K. \\ ${ }^{5}$ Ain Shams University, Faculty of Science, Cairo 11566, Egypt.
}

\begin{abstract}
We analyze the anomalous magnetic moment of the muon $a_{\mu}$ in supersymmetric scenarios. First we concentrate on scenarios with universal soft terms. We find that a moderate increase of $a_{\mu}$ can be obtained by lowering the unification scale $M_{G U T}$ to intermediate values $10^{10-12} \mathrm{GeV}$. However, large values of $\tan \beta$ are still favored. Then we study the case of non-universal soft terms. For the usual value $M_{G U T} \approx 10^{16}$ $\mathrm{GeV}$, we obtain $a_{\mu}$ in the favored experimental range even for moderate $\tan \beta$ regions $(\tan \beta \gtrsim 5)$. Finally, we give an explicit example of these scenarios. In particular, we show that in a D-brane model, where the string scale is naturally of order $10^{10-12}$ $\mathrm{GeV}$ and the soft terms are non universal, $a_{\mu}$ is enhanced with low $\tan \beta$.
\end{abstract}

PACS: 14.60.Ef, 12.60.Jv, 11.25.Mj

Keywords: muon magnetic moment, scales, supersymmetry, superstrings. 


\section{Introduction}

Recently, an intense theoretical activity about new physics contributions to the anomalous magnetic moment of the muon $\left(a_{\mu}\right)$ has appeared in the literature [1]-[7]. This has been motivated by the new measurement in the E821 experiment at the Brookhaven National Laboratory (BNL) [8], where a $2.6 \sigma$ deviation from the standard model (SM) predictions [1], 2] was reported

$$
a_{\mu}(\mathrm{E} 821)-a_{\mu}(\mathrm{SM})=(43 \pm 16) \times 10^{-10} .
$$

There are also criticisms about the SM prediction quoted by this experiment [5]| It is not impossible that this deviation is due to the hadronic contribution to the vacuum polarization [2], which is the largest source of error reflecting the large experimental uncertainty of the data. However, the possibility that new physics effects are at the origin of the BNL deviation is very exciting. First of all, the new physics scale should be pretty close to the electroweak threshold, since its contributions to $a_{\mu}$ are of the same order or even larger than the corresponding electroweak corrections [1]. Besides, it has to pass all the electroweak precision tests of the SM and must be in agreement with all the known results from accelerators experiments.

The minimal supersymmetric standard model (MSSM) is a well established candidate for such a theoryll. On a general ground, if the MSSM is responsible of the BNL deviation, then the supersymmetric (SUSY) particle spectrum should be in the expected discovery range of Fermilab $2 \mathrm{TeV} p \bar{p}$ collider and certainly of the Large Hadronic $p p$ Collider (LHC) at CERN.

The most popular SUSY-breaking scenarios in the MSSM have been recently reanalyzed in the light of the new BNL results [3]. In particular, in the supergravity scenario the main conclusions can be summarized as follows. The requirement that the SUSY contribution to $a_{\mu}$ is within the $2 \sigma$ level in eq.(1), leads to the following upper limits on the lightest chargino and neutralino mass, respectively $m_{\chi^{ \pm}} \lesssim 600 \mathrm{GeV}$ and $m_{\chi^{0}} \lesssim 300 \mathrm{GeV}$ for $\tan \beta \leq 30$. The corresponding upper bound on the sneutrino masses is weaker and of the order of $1 \mathrm{TeV}[7]$.

The above analysis was performed assuming universality of the soft-breaking terms at the unification scale, $M_{G U T} \approx 10^{16} \mathrm{GeV}$, as is usually done in the MSSM literature. As known, such a scale can be obtained in the superstring framework, in particular this is the case of weakly coupled heterotic string [9], type I string [10, 11] and heterotic Mtheory [10, 12]. However, recently, it was realized that the string scale may be anywhere

\footnotetext{
${ }^{*}$ It is worth noticing that a recent paper [6] refutes these arguments.

${ }^{\dagger}$ see ref. . for alternative possibilities which might explain this deviation, such as leptoquarks, compositness, large extra dimensions models, etc.
} 
between the weak and the Plank scale. For instance, D-brane configurations where the SM lives, allow these possibilities in type I strings [13]-17], and similar results can also be obtained in type II strings [18 as well as in strongly and weakly coupled heterotic strings [19, 20].

To use the value of the initial scale, say $M_{I}$, as a free parameter for the running of the soft terms is particularly interesting since there are several arguments in favor of SUSY scenarios with scales $M_{I} \approx 10^{10-14} \mathrm{GeV}$. First, these scales were suggested in [19] to explain many experimental observations as neutrino masses or the scale for axion physics. Second, with the string scale of order $10^{10-12} \mathrm{GeV}$ one is able to attack the hierarchy problem of unified theories without invoking any hierarchically suppressed non-perturbative effect [16]. Third, for intermediate scale scenarios, charge and color breaking constraints become less important [21]. Let us recall that, due to these constraints, when working with the usual unification scale, $M_{G U T} \approx 10^{16}$ $\mathrm{GeV}$, there are extensive regions in the parameter space of soft SUSY-breaking terms that become forbidden [22]. There are other arguments in favor of scenarios with initial scales $M_{I}$ smaller than $M_{G U T}$. For example these scales might also explain the observed ultra-high energy $\left(\approx 10^{20} \mathrm{eV}\right)$ cosmic rays as products of long-lived massive string mode decays. Besides, several models of chaotic inflation favor also these scales [23. Finally, D-brane models lead naturally to intermediate values for the string scale, in order to reproduce low-energy data 17.

Inspired by these scenarios, it was recently pointed out that the neutralino-nucleon cross sections, which are relevant for dark matter experiments, are very sensitive to the variation of the initial scales for the running of the soft SUSY-breaking terms 24, 25, 17]. In particular, it was found that the smaller the scale is the larger the cross sections become. For instance, by taking $10^{10-12} \mathrm{GeV}$ rather than $M_{G U T}$, extensive regions in the parameter space of the MSSM have been found [24] where the neutralinonucleon cross sections are in the expected range of sensitivity of DAMA 26] and CDMS 27] detectors, and this even for moderate $\tan \beta$ regions $(\tan \beta \geq 3)$. This analysis was performed in the universal scenario for the soft terms. In contrast, in the usual case with initial scale at $M_{G U T}$, these large cross sections are achieved only for $\tan \beta>20$ 28, 29.

The fact that smaller initial scales imply larger neutralino-nucleon cross sections can be basically understood as follows. These cross sections are very sensitive to the $\mu$ parameter, which is the standard coupling in the superpotential between the two Higgs doublets, since they increase when $\mu$ decreases. Furthermore, the value of $\mu$ is also very sensitive to the initial scale $M_{I}$ and it decreases when $M_{I}$ decreases. As a consequence, decreasing $M_{I}$ one obtains larger cross sections [24]. 
One of the purposes of the present paper is to analyze, in the light of the new BNL results and in connection to the work of ref. [24], the variations of $a_{\mu}$ as a function of the initial scale $M_{I}$. The main reason is that in the MSSM $a_{\mu}$ is expected to be particularly sensitive to the $\mu$ parameter and therefore to the initial scale.

On the other hand, the soft SUSY-breaking terms can have in general a nonuniversal structure in the MSSM. Such non-universality can be derived from supergravity and superstring models [31]. In fact, it was shown in ref.[28] that non-universal scenarios allow for a remarkable enhancement of the neutralino-nucleon cross section to be in the current experimental regions, and this even for $\tan \beta>4$. Here and along this line, we will analyze the effect induced on $a_{\mu}^{\text {SUSY }}$ by the non-universality of the soft terms.

Finally, we give an explicit example where both situations, non-universal soft terms and an intermediate scale, are realized. This is the case of a D-brane model.

The paper is organized as follows. In section 2 we review general formulae for the SUSY contributions to $a_{\mu}$. In section 3 we study the prediction for $a_{\mu}^{\text {SUSY }}$ in SUSY scenarios with universal soft terms, when intermediate scales are allowed. Section 4 is devoted to the study of the effect of the non-universality of the soft terms on $a_{\mu}^{\mathrm{SUSY}}$. This is carried out first in the context of the MSSM with the usual scale $M_{G U T} \approx 10^{16}$ $\mathrm{GeV}$, and second in the framework of D-brane constructions. The conclusions are given in section 5 .

\section{SUSY contributions to the muon anomalous mag- netic moment}

The supersymmetric contributions to $a_{\mu}$ are mainly via magnetic-dipole penguin diagrams with an exchange of sneutrino-chargino or smuon-neutralino in the loop. These contributions can be found in the literature [32]-[35], and they are given by:

$$
\begin{aligned}
a_{\mu}^{\chi^{0}} & =\frac{m_{\mu}}{16 \pi^{2}} \sum_{m, i}\left\{-\frac{m_{\mu}}{6 m_{\tilde{\mu}_{m}}^{2}\left(1-x_{m i}\right)^{4}}\left(\left|N_{m i}^{L}\right|^{2}+\left|N_{m i}^{R}\right|^{2}\right)\right. \\
& \times\left(1-6 x_{m i}+3 x_{m i}^{2}+2 x_{m i}^{3}-6 x_{m i}^{2} \ln x_{m i}\right) \\
& \left.+\frac{m_{\chi_{i}^{0}}}{m_{\tilde{\mu}_{m}}^{2}\left(1-x_{m i}\right)^{3}} N_{m i}^{L} N_{m i}^{R}\left(1-x_{m i}^{2}+2 x_{m i} \ln x_{m i}\right)\right\} \\
a_{\mu}^{\chi^{ \pm}} & =\frac{m_{\mu}}{16 \pi^{2}} \sum_{k}\left\{\frac{m_{\mu}}{3 m_{\tilde{\nu}}^{2}\left(1-x_{k}\right)^{4}}\left(\left|C_{k}^{L}\right|^{2}+\left|C_{k}^{R}\right|^{2}\right)\right.
\end{aligned}
$$




$$
\begin{aligned}
& \times\left(1+1.5 x_{k}+0.5 x_{k}^{3}-3 x_{k}^{2}+3 x_{k} \ln x_{k}\right) \\
& \left.-\frac{3 m_{\chi_{k}^{ \pm}}}{m_{\tilde{\nu}}^{2}\left(1-x_{k}\right)^{3}} C_{k}^{L} C_{k}^{R}\left(1-\frac{4 x_{k}}{3}+\frac{x_{k}^{2}}{3}+\frac{2}{3} \ln x_{k}\right)\right\},
\end{aligned}
$$

where $x_{m i}=m_{\chi_{i}^{0}}^{2} / m_{\tilde{\mu}_{m}}^{2}, x_{k}=m_{\chi_{k}^{ \pm}}^{2} / m_{\tilde{\nu}}^{2}$,

$$
\begin{aligned}
N_{m i}^{L} & =h_{\mu}\left(U_{\chi^{0}}\right)_{3 i}\left(U_{\tilde{\mu}}\right)_{L m}+\sqrt{2} g_{1}\left(U_{\chi^{0}}\right)_{1 i}\left(U_{\tilde{\mu}}\right)_{R m}, \\
N_{m i}^{R} & =-h_{\mu}\left(U_{\chi^{0}}\right)_{3 i}\left(U_{\tilde{\mu}}\right)_{R m}+\frac{g_{2}}{\sqrt{2}}\left(U_{\chi^{0}}\right)_{2 i}\left(U_{\tilde{\mu}}\right)_{L m}+\frac{g_{1}}{\sqrt{2}}\left(U_{\chi^{0}}\right)_{1 i}\left(U_{\tilde{\mu}}\right)_{L m}, \\
C_{k}^{L} & =h_{\mu} U_{k 2}, \\
C_{k}^{R} & =-g_{2} V_{k 1} .
\end{aligned}
$$

Here $\left(U_{\chi^{0}}\right)_{i j}$ with $i, j=1,4,\left(U_{\tilde{\mu}}\right)_{(R, L) m}$ with $m=1,2$, and $U_{k l}, V_{k l}$ with $k, l=1,2$ are the neutralino, smuon and chargino mixing matrices respectively, $m_{\chi_{i}^{0}}, m_{\tilde{\mu}_{m}}, m_{\tilde{\nu}}$ and $m_{\chi_{k}^{ \pm}}$are the neutralino, smuon, sneutrino and chargino mass eigenstates respectively, $m_{\mu}$ is the muon mass, $h_{\mu}$ is the Yukawa coupling of the muon and $g_{i}$ are the electroweak gauge couplings. Let us remark that we are using the following sign conventions for Yukawa couplings, and gaugino and Higgsino masses in the Lagrangian: $\mathcal{L}=-h_{u} H_{u}^{0} \bar{u}_{L} u_{R}-h_{d} H_{d}^{0} \bar{d}_{L} d_{R}-h_{e} H_{d}^{0} \bar{e}_{L} e_{R}+\frac{1}{2} \sum_{a} M_{a} \lambda_{a} \lambda_{a}+\mu \tilde{H}_{u}^{0} \tilde{H}_{d}^{0}+$ h.c., with the neutralino basis given by $\left(\tilde{B}^{0}=-i \lambda^{\prime}, \tilde{W}_{3}^{0}=-i \lambda^{3}, \tilde{H}_{u}^{0}, \tilde{H}_{d}^{0}\right)$.

Eqs.(2-4) show that the dominant contributions to $a_{\mu}^{\chi^{ \pm}}$and $a_{\mu}^{\chi^{0}}$ correspond to the terms with $C^{L} C^{R}$ and $N^{L} N^{R}$ [33], since they are proportional to the chargino and neutralino masses respectively. Furthermore, it was found that the chargino contribution dominates the neutralino contribution [33]. Note e.g. that the lightest neutralino $\chi_{1}^{0}$ is often bino-like, i.e. $\left(U_{\chi^{0}}\right)_{11} \sim 1$ and $\left(U_{\chi^{0}}\right)_{1 i}<<1$ for $i=2,3,4$. Therefore the terms proportional to $g_{1}^{2}$ are expected to give the dominant contribution to $a_{\mu}^{\chi^{0}}$. However, these terms are always suppressed by the matrix entries $\left(U_{\tilde{\mu}}\right)_{L 2}$ or $\left(U_{\tilde{\mu}}\right)_{R 1}$ which are of order $m_{\mu} / m_{S U S Y}$. On the contrary, the chargino contribution $C^{L} C^{R}$ do not have such a suppression in the chargino mixing.

Eqs.(2-4) also show that $a_{\mu}^{\text {SUSY }}$ becomes larger as $\tan \beta$, the ratio of Higgs vacuum expectation values $\left\langle H_{2}\right\rangle /\left\langle H_{2}\right\rangle$, increases [36, 32]. Recall in this sense that for tan $\beta$ not too small the dominant chargino contribution can be approximated as [35]

$$
a_{\mu}^{\chi^{ \pm}} \approx \frac{3 \alpha_{2}}{4 \pi} \tan \beta \frac{m_{\mu}^{2} \mu M_{2}}{m_{\tilde{\nu}}^{2}\left(M_{2}^{2}-\mu^{2}\right)}\left[f\left(x_{M_{2}}\right)-f\left(x_{\mu}\right)\right]
$$

where $x_{M_{2}}=M_{2}^{2} / m_{\tilde{\nu}}^{2}, x_{\mu}=\mu^{2} / m_{\tilde{\nu}}^{2}, M_{2}$ is the weak gaugino mass and $f$ is a loop function defined as

$$
f(x)=\frac{3-4 x+x^{2}+2 \log (x)}{3(1-x)^{3}} .
$$


This approximate formula helps also to draw some important conclusions on the SUSY contributions to $a_{\mu}$, which we have checked that are still valid for low $\tan \beta$ as well.

First, decreasing the values of $M_{2}, \mu$ and $m_{\tilde{\nu}}^{2}$ leads to increase $a_{\mu}^{\text {SUSY }}$. Indeed, different scenarios that enhance $a_{\mu}^{\text {SUSY }}$ (as we will show in next sections) are based on the decrease of these quantities.

Second, The sign of $a_{\mu}^{\text {SUSY }}$ is given by the sign of the product $\mu M_{2}$ since the factor $\left(f\left(x_{M_{2}}\right)-f\left(x_{\mu}\right)\right) /\left(M_{2}^{2}-\mu^{2}\right)$ is positive in general. Assuming $M_{2}$ is real and positive (after performing $U(1)_{R}$ rotation), the positiveness of $a_{\mu}^{\mathrm{SUSY}}$ implies that $\mu$ should be positive. This has interesting consequences for the $b \rightarrow s \gamma$ constraints (at large $\tan \beta$ ) and also for the dark matter detection rate. It is known that for $\mu<0$ the neutralinonucleon cross section is reduced a lot due to accidental cancellations between different contributions. Also experimental constraints coming from the $b \rightarrow s \gamma$ process highly reduce the $\mu<0$ parameter space.

Third, as it is known, in order to satisfy the Higgs mass bound $\left(m_{H} \gtrsim 114 \mathrm{GeV}\right)$ large stop masses are required to increase the radiative corrections to the Higgs mass. However, as mentioned above, large values of $a_{\mu}^{\text {SUSY }}$ require light sneutrino and smuon. Thus a non-universal pattern of the soft SUSY-breaking terms would be preferred to fulfil both conditions. In particular, a pattern with light sleptons and heavy squarks.

Fourth, the trilinear coupling $A$ appears in left-right smuon mixing, following our sign conventions discussed below eq.(四), as $m_{\mu}(A-\mu \tan \beta)$ and stop mixing as $m_{\text {top }}(A-$ $\mu \cot \beta)$. It has a significant effect on the stop mass and large values of $|A|$ are favored. On the contrary, the smuon mixing is dominated by the $\mu$ term (specially in the large $\tan \beta$ region). However, for low $\tan \beta$ (and also low $\mu$ ) $A$-terms could have important effects if $A \simeq \mathcal{O}(-3 m)$.

\section{$3 a_{\mu}$ in SUSY models with intermediate scale}

In this section we consider the predictions for $a_{\mu}$ in the MSSM as a function of the initial scale $M_{I}$ for the soft SUSY-breaking terms. As discussed in the Introduction, there are very interesting arguments in favour of scenarios with scales $M_{I}$ smaller than $M_{G U T}$. Following the analysis of ref. 24] we will consider two possible scenarios with "intermediate" initial scales, concerning the unification of the gauge couplings.

First, we will assume that these are non universal and their values will depend on the initial scale $M_{I}$ chosen. For instance, for the scale $M_{I}=10^{11} \mathrm{GeV}$, one obtains $g_{3} \approx 0.8, g_{2} \approx 0.6$ and $g_{1} \approx 0.5$. This scenario might be inspired for example by D- 
brane configurations where the SM lives. If the SM comes from the same collection of D-branes, stringy corrections might change the boundary conditions at the string scale $M_{I}$ in order to mimic the effect of field theoretical logarithmic running [11, 30]. Another possibility giving rise to a similar result might arise when the gauge groups came from different types of D-branes. Since different D-branes have associated different couplings, this would imply the non universality of the gauge couplings (see ref. [17] and references therein).

On the other hand, to obtain gauge coupling unification at $M_{I}, \alpha_{i}=\alpha$, is possible with the addition of extra fields in the massless spectrum [16]. An example of additional particles which can produce the beta functions, $b_{3}=-3, b_{2}=3, b_{1}=19$, yielding unification at around $M_{I}=10^{11} \mathrm{GeV}$ was given in ref.[21], $2 \times[(1,2,1 / 2)+$ $(1,2,-1 / 2)]+3 \times[(1,1,1)+(1,1,-1)]$, where the fields transform under the SM gauge group. In this example one has $g\left(M_{I}\right) \approx 0.8$.

It was obtained in ref. [24] that, due to the different values of the gauge couplings at $M_{I}$, the above scenarios give rise to qualitatively different results for neutralino-nucleon cross sections. In this section we will also analyze this issue for $a_{\mu}$.

Let us concentrate first on the scenario with non-universal gauge couplings at $M_{I}$. We assume, as in the minimal supergravity scenario, universality in the soft-breaking sector. As usual, we eliminate the free parameter $\mu$ which appears in the superpotential $W=-\mu H_{1}^{0} H_{2}^{0}$, by requiring the correct electroweak breaking at the $M_{Z}$ scale. These requirements leave us with the following independent parameters at the initial scale $M_{I}: m, M_{1 / 2}, A, \tan \beta$, and the sign $(\mu)$, respectively the common scalar mass, gaugino mass, the coefficient of trilinear terms, and the ratio of Higgs vacuum expectation values. Let us finally remark that we are assuming gaugino mass universality at the high energy scale, although in this scenario gauge couplings do not unify. This situation is in principle possible in generic supersymmetric models, however it is not so natural in supersymmetric models from supergravity where gaugino masses and gauge couplings are related through the gauge kinetic function. Since an explicit string construction with nonuniversal gauge couplings and gaugino masses will be analyzed in detail in Subsection 4.2, we choose to simplify the discussion here assuming gaugino mass universality.

As emphasized in ref.[24], lowering the unification scale decreases the value of $\mu$. Thus we will analyze here what is the influence of this decrease on $a_{\mu}^{\text {SUSY }}$. Let us write eq.(5) as

$$
a_{\mu}^{\chi^{ \pm}} \approx \frac{3 \alpha_{2}}{4 \pi} \tan \beta m_{\mu}^{2} x_{\mu}^{1 / 2} x_{M_{2}}^{1 / 2} F\left(x_{M_{2}}, x_{\mu}\right),
$$

where $F\left(x_{M_{2}}, x_{\mu}\right)=\left(f\left(x_{M_{2}}\right)-f\left(x_{\mu}\right)\right) /\left(M_{2}^{2}-\mu^{2}\right)$ is a function which depends on $\mu, M_{2}$ 

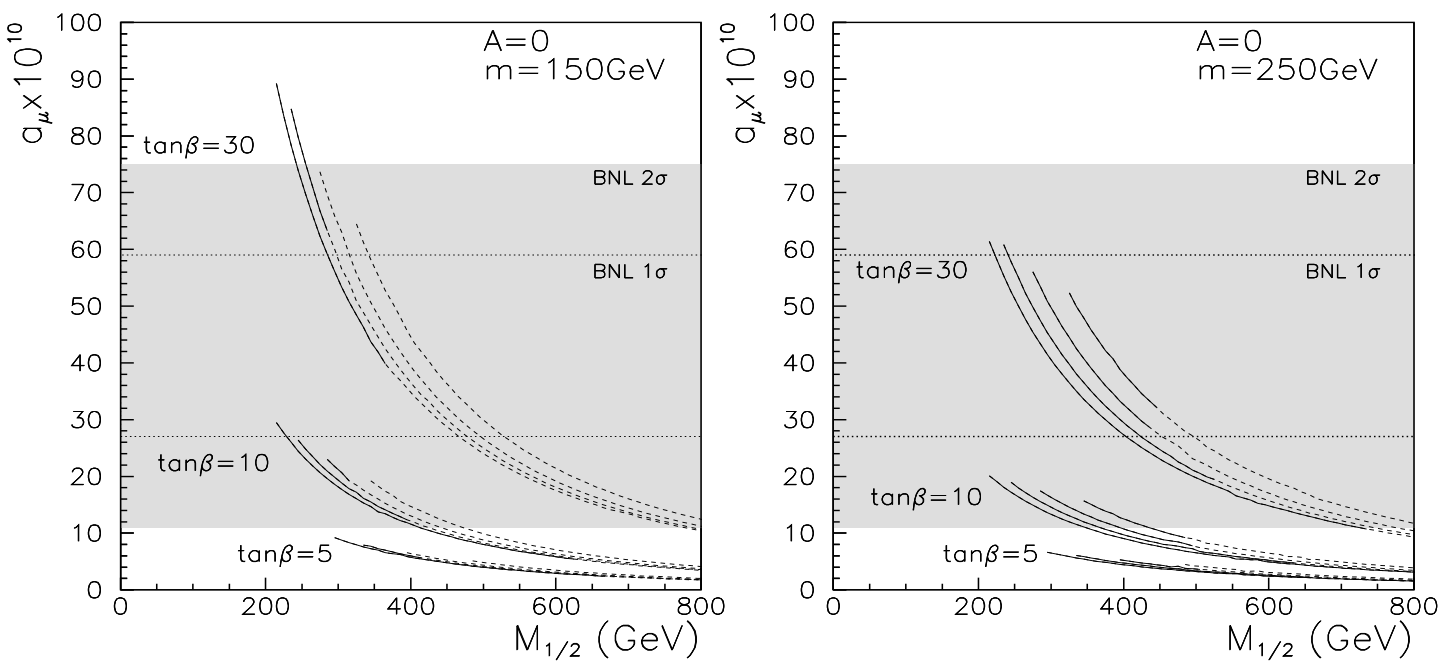

Figure 1: $\quad a_{\mu}^{\mathrm{SUSY}}$ as a function of the common gaugino mass $M_{1 / 2}$. The four curves inside each set associated to a particular value of $\tan \beta$ correspond, from bottom to top, to $M_{I}=10^{16,14,12,10} \mathrm{GeV}$ respectively. Continuous lines correspond to regions where the neutralino is the LSP.

and $m_{\tilde{\nu}}$. It turns out that when we lower the scale, the variation of $\mu$ is much more important than the variation of $M_{2}$ and $m_{\tilde{\nu}}$. Although this produces an important decrease in $x_{\mu}$ (while the increase in $x_{M_{2}}$ is moderate), the big increase in $F$ compensates it. In this way, higher values of $a_{\mu}^{\mathrm{SUSY}}$ can be obtained.

We recall that low initial scales play a crucial role in increasing the spin-independent part of the neutralino-nucleon cross sections, mainly due to the decrease of the $\mu$ parameter [24]. In the MSSM with universal scenario at $M_{G U T}$ these cross sections are strongly suppressed due to the fact that the lightest neutralino is mainly Bino. By decreasing the value of the $\mu$ parameter, the Higgsino components of the lightest neutralino increase and therefore also the spin-independent part of the cross sections increases. On the contrary, the sensitivity of $a_{\mu}^{\text {SUSY }}$ versus the initial scale is quite moderate.

We show the results of our analysis in Figs. 11 and 2. They have been obtained using the general formulae (2-4) discussed in Section 2. These figures correspond to the $\mu>0$ case. We have not included the scenarios with opposite values of $\mu$ since they imply negative values for $a_{\mu}^{\text {SUSY }}$ and therefore are ruled out by the BNL results.

In Fig. 11 we plot $a_{\mu}^{\mathrm{SUSY}}$ versus the common gaugino mass at the initial scale, $M_{1 / 2}$, for a fixed value of $m=150,250 \mathrm{GeV}$, and $A=0$. Inside each plot there are three sets of four curves which correspond to $\tan \beta=5,10,30$. The four curves inside each set correspond to $M_{I}=10^{16,14,12,10} \mathrm{GeV}$, from bottom to top respectively, and 


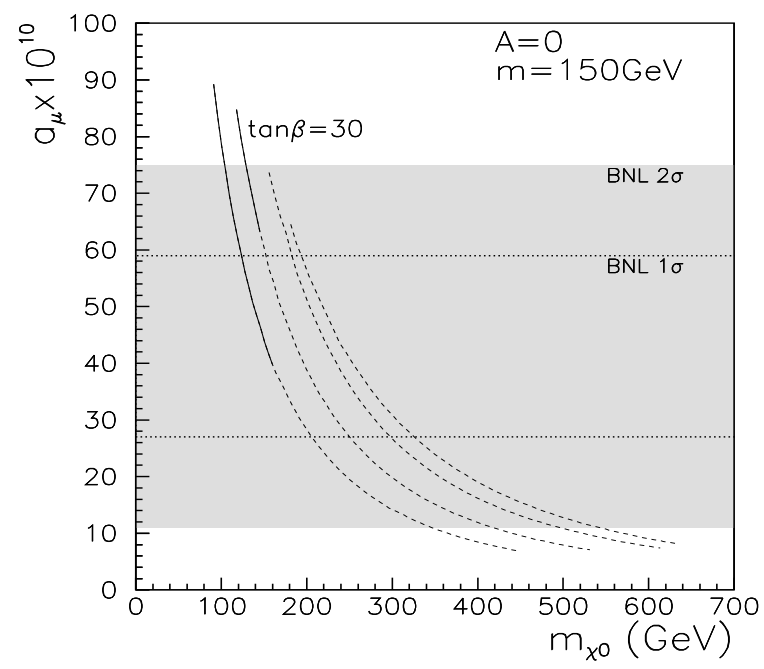

Figure 2: $a_{\mu}^{\text {SUSY }}$ as a function of the lightest neutralino mass $m_{\chi^{0}}$. The four curves correspond, from bottom to top, to $M_{I}=10^{16,14,12,10} \mathrm{GeV}$ respectively.

the continuous lines correspond to regions where the neutralino is the lightest SUSY particle (LSP). Finally, the large grey area stands for the BNL deviation at $2 \sigma$ level. We have checked that our results are consistent with present bounds coming from accelerators. These are LEP and Tevatron bounds on supersymmetric masses and CLEO $b \rightarrow s \gamma$ branching ratio measurements. The former are the reason why regions with $M_{1 / 2} \lesssim 200 \mathrm{GeV}$ are not allowed. In particular, the Higgs mass bound $\left(m_{H} \gtrsim 114\right.$ $\mathrm{GeV})$ is not fulfilled. Although not shown in the figure, $b \rightarrow s \gamma$ results constrain the value of $a_{\mu}^{\text {SUSY }} \times 10^{10}$ to be smaller than about $60(45)$ for $\tan \beta=30$ in the case $m=150(250) \mathrm{GeV}$.

As shown in Fig. 1, for a given $\tan \beta$ the smaller the scale is, the larger $a_{\mu}^{\text {SUSY }}$ becomes. We have checked that the dominant contribution is due to the chargino, as discussed in the previous section. For example, for $\tan \beta=30$, the neutralino contribution is not only small but also decreases going from $M_{G U T}$ to $10^{10} \mathrm{GeV}$ and becomes negative. In any case, as mentioned above, the sensitivity of $a_{\mu}^{\mathrm{SUSY}}$ to the scale is quite moderate.

On the other hand, as discussed in the previous section, we obtain that $a_{\mu}^{\mathrm{SuSY}}$ increases with $\tan \beta$. Besides, also the deviation with the scale in $a_{\mu}^{\mathrm{SUSY}}$ increases with $\tan \beta$. By comparing the plots with $m=150 \mathrm{GeV}$ and $m=250 \mathrm{GeV}$ we see that $a_{\mu}^{\text {SUSY }}$ decreases when $m$ increases, due to the fact that the smuon and sneutrino become heavier, but for fixed $\tan \beta$ the scale dependence in $a_{\mu}^{\mathrm{SUSY}}$ remains essentially the same.

Thus the main conclusion drawn from the results in Fig. 1 is that, within the $2 \sigma$ 

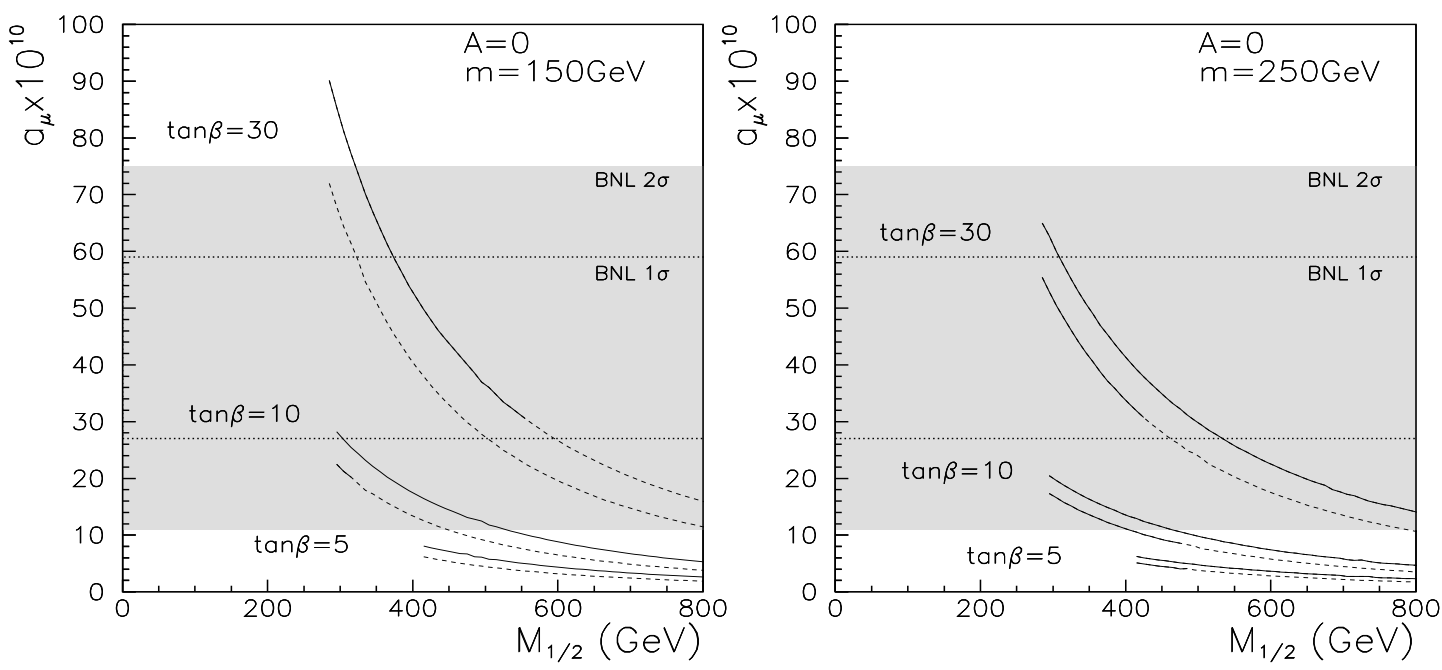

Figure 3: $\quad a_{\mu}^{\text {SUSY }}$ as a function of the common gaugino mass $M_{1 / 2}$ for $M_{I}=3 \times 10^{11} \mathrm{GeV}$. The two curves inside each set associated to a particular value of $\tan \beta$ correspond, from bottom to top, to the cases without and with gauge couplings unification, respectively. Continuous lines correspond to regions where the neutralino is the LSP.

level of the BNL deviation, the low $\tan \beta$ regions (namely $\tan \beta \leq 5$ ) are excluded for any scale in the range $M_{I}=10^{10-16} \mathrm{GeV}$. Besides, the sensitivity to the scale is quite moderate even for large $\tan \beta$. Note however that for $M_{1 / 2}$ between 350 and $450 \mathrm{GeV}$ and $\tan \beta=10$ whereas the value of $a_{\mu}$ is in the forbidden region for $M_{G U T}$, it is in the allowed region for intermediate scales.

The plots in Fig. 1 1 have been obtained for $A=0$. However, we have checked that $a_{\mu}^{\mathrm{SUSY}}$ is quite insensitive to this choice, in particular, taking $A= \pm M_{1 / 2}$ the corresponding results are slightly modified.

In Fig. 2 we show the dependence of $a_{\mu}^{\text {SUSY }}$ versus the lightest neutralino mass varying the scale in the same range of Fig. 1, for the representative case of $\tan \beta=30$ and $m=150 \mathrm{GeV}$. By requiring that $a_{\mu}^{\mathrm{SUSY}}$ is within the lower bound of the $2 \sigma \mathrm{BNL}$ region in Fig. 2, the following upper bounds for the SUSY mass can be obtained: $m_{\chi^{0}} \leq 340(540) \mathrm{GeV}$ with $M_{I}=10^{16}\left(10^{10}\right) \mathrm{GeV}$.

The same analysis can be carried out for the lightest chargino, smuon and sneutrino with the result $m_{\chi^{ \pm}} \leq 640(560) \mathrm{GeV}, \quad m_{\tilde{\mu}} \leq 330(300) \mathrm{GeV}$, and $m_{\tilde{\nu}} \leq 560(460) \mathrm{GeV}$. Smaller (and therefore less conservative) upper bounds can be obtained by taking smaller values of $\tan \beta$ and/or larger values of the common scalar mass $m$.

Let us consider now the case with gauge coupling unification at $M_{I}$ mentioned above. This scenario is analyzed in Fig. 3 where $a_{\mu}^{\mathrm{SUSY}}$ is plotted versus $M_{1 / 2}$ for 
$\tan \beta=5,10,30$, and $m=150,250 \mathrm{GeV}$. Each set of curves show also the case without gauge unification (lower one) studied above for comparison with the case with gauge unification (upper one), both for $M_{I}=3 \times 10^{11} \mathrm{GeV}$.

From the plots in Fig. 3 one can learn that in the case with unification the contribution to $a_{\mu}^{\mathrm{SUSY}}$ is increased with respect to the case without unification. The reason being that now $\alpha_{2}\left(M_{I}\right)$ is bigger and therefore the weak gaugino mass $M_{2}$ is smaller at low energy. This is the opposite to what happens in the case of dark matter analyses. There, in the case with gauge unification the neutralino-nucleon cross sections are decreased. It is worth remarking that in Fig. 3 it is the lower bound on the Higgs mass, that we set to be $m_{H} \gtrsim 114 \mathrm{GeV}$, which prevents the common gaugino mass $M_{1 / 2}$ from taking lower values than about $300 \mathrm{GeV}$.

\section{$4 a_{\mu}$ in SUSY models with non-universal soft terms}

\subsection{MSSM with non-universality}

As mentioned in the Introduction, the soft SUSY-breaking terms can have in general a non-universal structure in the MSSM. Here we will analyze the effect induced by this non-universality on $a_{\mu}^{\mathrm{SUSY}}$. In particular we will see that parameterizations of the soft terms producing an enhancement of $a_{\mu}^{\mathrm{SUSY}}$ are possible, making it within the experimental limit even with low $\tan \beta$, unlike the universal cases.

The SUSY contributions to $a_{\mu}^{\mathrm{SUSY}}$ depend essentially on the gaugino masses $M_{i}$, the slepton masses $m_{l_{L}}^{2}, m_{e_{R}}^{2}$, and the values of $\mu, A_{\mu}$ and $\tan \beta$. As we discussed above, small $\mu, m_{\tilde{\nu}}, M_{2}$ are favored to enhance $a_{\mu}^{\mathrm{SUSY}}$. Therefore, here, we consider an scenario with non-universal soft breaking terms at $M_{G U T}$ where the sleptons and Higgs masses are parameterized by

$$
\begin{array}{lrl}
m_{H_{2}}^{2}=a m^{2}, & a>1, \\
m_{H_{1}}^{2}=m_{l_{L}}^{2}=m_{e_{R}}^{2}=b m^{2}, & 0 \leq b<1 .
\end{array}
$$

The squark masses, which are irrelevant for this analysis, are assumed to be universal and equal to $m$. Since the smaller(bigger) $m_{H_{1}}^{2}\left(m_{H_{2}}^{2}\right)$ at $M_{G U T}$ is, the less important the positive(negative) contribution to $\mu$ at the electroweak scale becomes, the above non-universality for Higgs masses will decrease the value of $\mu$. Reducing the soft slepton masses we also reduce the sneutrino and smuon masses. The gaugino masses are also assumed to be non-universal, as we will discuss below, and we have fixed $M_{2}$ such that the lightest chargino mass at the weak scale is of the order of the current experimental 


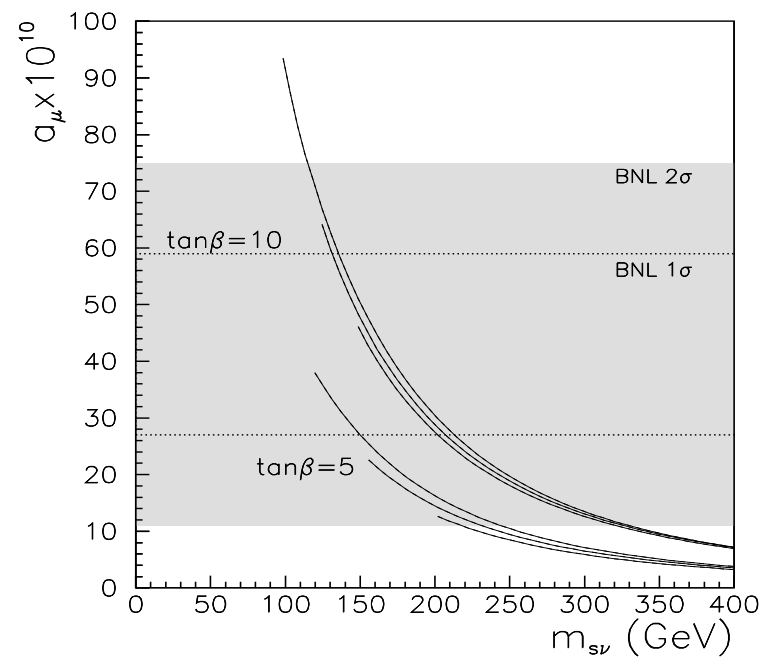

Figure 4: $a_{\mu}^{\text {SUSY }}$ as a function of the sneutrino mass $m_{\tilde{\nu}}$ in the MSSM with the nonuniversal soft terms discussed in the text. The three curves inside each set associated to a particular value of $\tan \beta$ correspond, from top to bottom, to $A=-3 m, 0,3 m$ respectively .

limit, i.e. $\mathcal{O}(100) \mathrm{GeV}$. Finally we assume that the A-terms are vanishing except for $A_{\mu}$.

We find that in this class of models, it is possible to obtain $a_{\mu}^{\mathrm{SUSY}}$ within the E821 $1 \sigma$ bounds with low $\tan \beta$. In Fig. $⿴$ we present the results for $a_{\mu}^{\text {SUSY }}$ as a function of the sneutrino mass for $\tan \beta=5$ and 10. We have assumed that $a=2, b=0.5$ and $\mathrm{m}$ varies from 150 to $600 \mathrm{GeV}$. We also fix $M_{1}=M_{2}=140 \mathrm{GeV}$ which leads to a lightest chargino mass of order $120 \mathrm{GeV}$ and a lightest neutralino mass of order $60 \mathrm{GeV}$. Also we need to take large values for $M_{3}$ of order $m$. In this particular example we use $M_{3}=\sqrt{3} m$. Three values for $A_{\mu}$ have been examined, namely $A_{\mu}=-3 m, 0,3 m$. As we can see from Fig. $⿴$ large and negative values of $A_{\mu}$ allow larger values of $a_{\mu}^{\mathrm{SUSY}}$.

It is worth noticing here that unlike the case with intermediate scales, now the neutralino contribution is positive, helping in increasing the value of $a_{\mu}^{\text {SUSY }}$. In any case, still the dominant contribution is due to the chargino.

It is remarkable that the non-universality of the soft SUSY-breaking terms has a very important role in enhancing the values of $a_{\mu}^{\text {SUSY }}$ and making it within the experimental limit even with low $\tan \beta$. In fact, the non-universality of the gaugino masses is crucial for such a enhancing. In the case of universal gaugino masses $M_{1}=$ $M_{2}=M_{3}=M_{1 / 2}$, the lower bound of the Higgs mass $\left(m_{H} \gtrsim 114 \mathrm{GeV}\right)$ requires large values of $M_{1 / 2}$. Recall that the Higgs mass gets a large contribution from the loop 
correction which is proportional to the stop mass. Since we want to have the lightest neutralino as the LSP we then need to push $m$ to higher values in order to avoid some slepton as the LSP. This, of course, leads to a heavy spectrum and hence $a_{\mu}^{\text {SUSY }}$ is supressed. Relaxing this assumption, we can have $M_{3}$ large in order to fulfil the Higgs bound, we can keep $M_{1}$ light to assure that the LSP is always the lightest neutralino, and we can have $m$ not very heavy. Furthermore, we found that if one assumes nonuniversality only in the gaugino sector, the enhancement of $a_{\mu}^{\mathrm{SUSY}}$ is not enough to be in the E821 regions. One still needs to assume that the slepton and Higgs masses are non-universal as discussed below eq.(8).

\subsection{D-brane models}

Recent studies of type I strings have shown that it is possible to construct a number of models with interesting phenomenological properties [37, 14, 38]. It was also shown that models with the gauge group and particle content of the supersymmetric standard model lead naturally to intermediate values for the string scale, in order to reproduce the value of gauge couplings deduced from experiments [17]. In addition, non-universal soft SUSY-breaking terms appear generically.

Type I models contain D-branes and the gauge groups of the SM may come from different types of D-branes or from the same type of D-branes. Although, as mentioned above, intermediate values for the string scale $\left(M_{I}=10^{10-12} \mathrm{GeV}\right)$ are naturally obtained we will consider here a model where also higher values are allowed. This will allow us to study the variations of $a_{\mu}$ as a function of the initial scale $M_{I}$, following the lines of Section 3.

In particular, in this model the gauge group $U(3) \times U(2) \times U(1)$, giving rise to $S U(3) \times S U(2) \times U(1)^{3}$, arises from three different types of D-branes, and therefore the gauge couplings will be non-universal. Interesting phenomenological properties of this model can be found in refs. [17, 40]. The analysis of the soft terms has been done under the assumption that only the dilaton $(S)$ and moduli $\left(T_{i}\right)$ fields contribute to SUSY breaking and it has been found that these soft terms are generically non-universal. Using the standard parameterization 39]

$$
\begin{aligned}
& F^{S}=\sqrt{3}\left(S+S^{*}\right) m_{3 / 2} \sin \theta \\
& F^{i}=\sqrt{3}\left(T_{i}+T_{i}^{*}\right) m_{3 / 2} \cos \theta \Theta_{i},
\end{aligned}
$$

where $i=1,2,3$ labels the three complex compact dimensions, and the angle $\theta$ and the $\Theta_{i}$ with $\sum_{i}\left|\Theta_{i}\right|^{2}=1$, just parametrize the direction of the goldstino in the $S, T_{i}$ field space, one is able to obtain the following soft terms [17. The gaugino masses are 
given by

$$
\begin{aligned}
& M_{3}=\sqrt{3} m_{3 / 2} \sin \theta \\
& M_{2}=\sqrt{3} m_{3 / 2} \Theta_{1} \cos \theta \\
& M_{Y}=\sqrt{3} m_{3 / 2} \alpha_{Y}\left(M_{I}\right)\left(\frac{2 \Theta_{3} \cos \theta}{\alpha_{1}\left(M_{I}\right)}+\frac{\Theta_{1} \cos \theta}{\alpha_{2}\left(M_{I}\right)}+\frac{2 \sin \theta}{3 \alpha_{3}\left(M_{I}\right)}\right)
\end{aligned}
$$

where

$$
\frac{1}{\alpha_{Y}\left(M_{I}\right)}=\frac{2}{\alpha_{1}\left(M_{I}\right)}+\frac{1}{\alpha_{2}\left(M_{I}\right)}+\frac{2}{3 \alpha_{3}\left(M_{I}\right)} .
$$

Here, relation (11) is due to the D-brane origin of the $U(1)$ gauge groups. In particular $U(1)_{Y}$ is a linear combination of the three $U(1)$ gauge groups arising from $U(3), U(2)$ and $U(1)$ within three different D-branes. $\alpha_{k}$ correspond to the gauge couplings of the $U(k)$ branes. As shown in Ref.[17], $\alpha_{1}\left(M_{I}\right)=0.1(1)$ leads to the string scale $M_{I}=10^{12}\left(5 \times 10^{15}\right) \mathrm{GeV}$.

The soft scalar masses are given by

$$
\begin{aligned}
m_{q}^{2} & =m_{3 / 2}^{2}\left[1-\frac{3}{2}\left(1-\Theta_{1}^{2}\right) \cos ^{2} \theta\right], \\
m_{d^{c}}^{2} & =m_{3 / 2}^{2}\left[1-\frac{3}{2}\left(1-\Theta_{2}^{2}\right) \cos ^{2} \theta\right], \\
m_{u^{c}}^{2} & =m_{3 / 2}^{2}\left[1-\frac{3}{2}\left(1-\Theta_{3}^{2}\right) \cos ^{2} \theta\right], \\
m_{e^{c}}^{2} & =m_{3 / 2}^{2}\left[1-\frac{3}{2}\left(\sin ^{2} \theta+\Theta_{1}^{2} \cos ^{2} \theta\right)\right], \\
m_{l}^{2} & =m_{3 / 2}^{2}\left[1-\frac{3}{2}\left(\sin ^{2} \theta+\Theta_{3}^{2} \cos ^{2} \theta\right)\right], \\
m_{H_{2}}^{2} & =m_{3 / 2}^{2}\left[1-\frac{3}{2}\left(\sin ^{2} \theta+\Theta_{2}^{2} \cos ^{2} \theta\right)\right], \\
m_{H_{1}}^{2} & =m_{l}^{2},
\end{aligned}
$$

and finally the trilinear parameters are

$$
\begin{aligned}
& A_{u}=\frac{\sqrt{3}}{2} m_{3 / 2}\left[\left(\Theta_{2}-\Theta_{1}-\Theta_{3}\right) \cos \theta-\sin \theta\right], \\
& A_{d}=\frac{\sqrt{3}}{2} m_{3 / 2}\left[\left(\Theta_{3}-\Theta_{1}-\Theta_{2}\right) \cos \theta-\sin \theta\right], \\
& A_{e}=0 .
\end{aligned}
$$

We observe that the angle $\theta$ and the $\Theta_{i}$ are quite constrained in order to avoid negative mass-squared for squarks and sleptons. This constraint allows a small region for the angle $\theta$, namely $0<\theta \lesssim \pi / 6$. From eq.(10), one notes that in this allowed region of $\theta$ we have at the string scale $M_{3}<M_{2}<M_{1}$. However, at the electroweak 

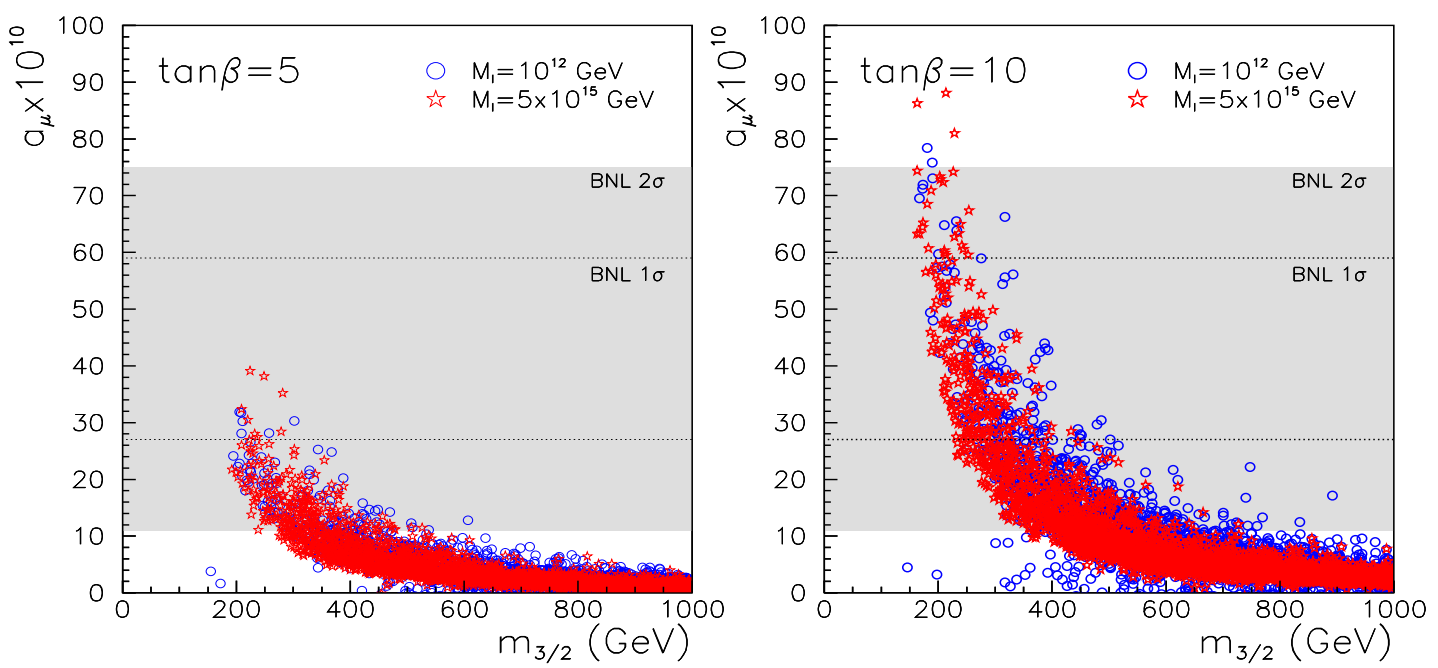

Figure 5: $a_{\mu}^{\mathrm{SUSY}}$ as a function of the gravitino mass $m_{3 / 2}$ in the D-brane model, for two values of the string scale $M_{I}=10^{12}$ and $5 \times 10^{15} \mathrm{GeV}$, and for $\tan \beta=5,10$.

scale and due to the different running we find that $M_{2}$ is the lightest gaugino mass, namely $M_{2}<M_{1}<M_{3}$. This is an interesting example for having the lightest chargino mass not very heavy and hence $a_{\mu}^{\text {SUSY }}$ could be enhanced as discussed in Section 2 .

In Fig. 5 we show a scatter plot of $a_{\mu}^{\text {SUSY }}$ as a function of the gravitino mass $m_{3 / 2}$ for a scanning of the parameter space discussed above. Two different values of $\tan \beta, 5$ and 10, are shown. Likewise, we consider the two values of the string scale discussed previously, $M_{I}=10^{12}$ and $5 \times 10^{15} \mathrm{GeV}$. Clearly, these models are much more constrained than the generic MSSM with non-universal soft terms. However, we can see that for a string scale of order $10^{12} \mathrm{GeV}$ the values of $a_{\mu}^{\text {SUSY }}$ are within the E821 $1 \sigma$ for $\tan \beta \gtrsim 5$. As expected from the discussion in Section 3 , smaller values of $a_{\mu}^{\text {SUSY }}$ are obtained for the scale $5 \times 10^{15} \mathrm{GeV}$.

\section{Conclusions}

In the light of the new BNL results on muon g-2, we have analyzed $a_{\mu}^{\text {SUSY }}$ in SUSY scenarios. First we have concentrated on scenarios with universal soft terms. In particular, we have analyzed the sensitivity of $a_{\mu}^{\text {SUSY }}$ with respect to the initial scales $M_{I}$, smaller than $M_{G U T}$, where soft SUSY-breaking terms are generated.

We have noted a moderate sensitivity of $a_{\mu}^{\text {SUSY }}$ to the value of the initial scales for the running of the soft terms. We found that the smaller the scale is the larger $a_{\mu}^{\text {SUSY }}$ becomes. In particular, by taking $M_{I} \approx 10^{10-12} \mathrm{GeV}$ rather than $M_{G U T} \approx 10^{16} \mathrm{GeV}$, 
which is a more sensible choice e.g. in the context of some superstring models, we find that $a_{\mu}^{\text {SUSY }}$ increases at most of $30 \%$ in the large $\tan \beta$ region $(\tan \beta=30)$, while it is less than $10 \%$ for $\tan \beta \leq 10$.

We applied the new BNL results to set upper bounds on the relevant SUSY spectrum, by requiring that $a_{\mu}^{\text {SUSY }}$ lies within the $2 \sigma$ BNL deviation. The main relevant result of BNL constraints is that the regions with $\tan \beta \leq 5$ are excluded for any scale $M_{I} \approx 10^{10-16} \mathrm{GeV}$. Besides, we have found upper bounds on the lightest neutralino, chargino, smuon, and sneutrino masses, namely of the order of $340,640,330,560 \mathrm{GeV}$ respectively, (for $\tan \beta=30$ and $m=150$ ) at $M_{I}=10^{16} \mathrm{GeV}$. These bounds are increased of $\mathcal{O}(60 \%)$ in the case of neutralino and are decreased of order $\mathcal{O}(10-20 \%)$ for the other ones, by decreasing the initial scale $M_{I}$ from $10^{16} \mathrm{GeV}$ to $10^{10} \mathrm{GeV}$.

We have also analyzed the corresponding results for the case of gauge couplings unification at intermediate scale $M_{I}=10^{11} \mathrm{GeV}$. In this case we have found that the values of $a_{\mu}^{\text {SUSY }}$ are higher, at most of $30 \%$, with respect to the corresponding ones mentioned above, with the same initial scale but without gauge couplings unification.

Then we have studied the possibility of having non-universal soft terms, which is a generic situation in supergravity and superstring models. For the usual scale $M_{G U T} \approx 10^{16} \mathrm{GeV}$, we have obtained $a_{\mu}$ in the favored experimental range even for moderate $\tan \beta$ regions ( $\tan \beta \gtrsim 5$ ). Obviously, from the previous result we can deduce that lowering the initial scale for the running of the non-universal soft terms, larges values for $a_{\mu}^{\text {SUSY }}$ will be obtained.

Finally, we have given an explicit example where the two situations discussed above occur. This is the case of a D-brane model, where the string scale is naturally of order $10^{10-12} \mathrm{GeV}$ and the soft terms are non universal. We have obtained that $a_{\mu}$ is enhanced with low $\tan \beta$.

\section{Note added}

As this manuscript was prepared, ref.41] appeared. The authors discuss also the variation of $a_{\mu}$ with the initial scale, however, their analysis concentrates on the dilaton limit.

\section{Acknowledgments}

D.G. Cerdeño acknowledges the financial support of the Comunidad de Madrid through a FPI grant. The work of S. Khalil was supported by PPARC. The work of C. Muñoz was supported in part by the Ministerio de Ciencia y Tecnología, and the European Union under contract HPRN-CT-2000-00148. The work of E. Torrente-Lujan was supported in part by the Ministerio de Ciencia y Tecnología. 


\section{References}

[1] A. Czarnecki and W.J. Marciano, hep-ph/0102122.

[2] M. Davier, and A. Höcker, Phys. Lett. B435 (1998) 427; M. Davier, hepex/9912044; S. Eidelman and F. Jegerlehner, Z. Phys. C67, 585 (1995); K. Adel and F.J. Yndurain, Rev. Acad. Ciencias (Esp.), 92 (1998), hep-ph/9509378; J.A. Casas, C. López and F.J. Yndurain, Phys. Rev. 32, 736 (1985); S. Narison, hepph/0103199.

[3] E.A. Baltz and P. Gondolo, hep-ph0102147; J. Feng and K.T. Matchev, hepph/0102146; L. Everett, G.L. Kane, S. Rigolin, and L.T. Wang, hep-ph/0102145; T. Ibrahim, U. Chattopadhyay, and P. Nath, hep-ph/0102324; R. Arnowitt, B. Dutta, B. Hu and Y. Santoso, hep-ph/0102344; K. Choi, K. Hwang, S.K. Kang, K.Y. Lee, and W.Y. Song, hep-ph/0103048; A. Dedes and H. E. Haber, hepph/0102297; S. Martin and J.D. Wells, hep-ph/0103067; S. Komine, T. Moroi, and M. Yamaguchi, hep-ph/0103182; H. Baer, C. Balázs, J. Ferrandis, and X. Tata, hep-ph/0103280; D.F. Carvalho, J. Ellis, M.E. Gomez and S. Lola, hepph/0103256; K. Enqvist, E. Gabrielli and K. Huitu, hep-ph/0104174.

[4] D. Chakraverty, D. Choudhury, and A. Datta, hep-ph/0102180; K. Cheung, hepph/0102238; X. Calmet, H. Fritzsch and D. Holtmannspötter, hep-ph/0103012; M.B. Einhorn and J. Wudka, hep-ph/0103034; K. Cheung, C.-H. Chou and O.C.W. Kong hep-ph/0103183.

[5] F.J. Ynduráin, hep-ph/0102312.

[6] W.J. Marciano and B.L. Roberts, hep-ph/0105056.

[7] U. Chattopadhyay and P. Nath, hep-ph/0102157.

[8] H.N. Brownn et al., hep-ex/0102017.

[9] For a review, see: K.R. Dienes, Phys. Rept. 287 (1997) 447, and references therein.

[10] E. Witten, Nucl. Phys. B471 (1996) 135.

[11] L.E. Ibanez, C. Muñoz and S. Rigolin, Nucl. Phys. B553 (1999) 43.

[12] T. Banks and M. Dine, Nucl. Phys. B479 (1996) 173; D.G. Cerdeño and C. Muñoz, Phys. Rev. D61 (2000) 016001. 
[13] J. Lykken, Phys. Rev. D54 (1996) 3693; N. Arkani-Hamed, S. Dimopoulos and G. Dvali, Phys. Rev. Lett. B249 (1998) 262; I. Antoniadis, N. Arkani-Hamed, S. Dimopoulos and G. Dvali, Phys. Lett. B436 (1998) 263; I. Antoniadis and C. Bachas, Phys. Lett. B450 (1999) 83.

[14] G. Shiu and S.-H.H. Tye, Phys. Rev. D58 (1998) 106007; Z. Kakushadze and S.-H.H. Tye, Phys. Rev. D58 (1998) 126001.

[15] I. Antoniadis, E. Kiritsis and T.N. Tomaras, Phys. Lett. B486 (2000) 186.

[16] C. Burgess, L.E. Ibañez and F. Quevedo, Phys. Lett. B447 (1999) 257.

[17] D.G. Cerdeño, E. Gabrielli, S. Khalil, C. Muñoz and E. Torrente-Lujan, hepph/0102270, to appear in Nucl. Phys. B.

[18] I. Antoniadis and B. Pioline, Nucl. Phys. B550 (1999) 41.

[19] K. Benakli, Phys. Rev. D60 (1999) 104002.

[20] K. Benakli and Y. Oz, Phys. Lett. B472 (2000) 83; A. Gregori, hep-th/0005198.

[21] S.A. Abel, B.C. Allanach, F. Quevedo, L.E. Ibáñez and M. Klein, JHEP 0012 (2000) 026.

[22] For a review, see e.g.: C. Muñoz, hep-ph/9709329, and references therein.

[23] N. Kaloper and A. Linde, Phys. Rev. D59 (1999) 101303.

[24] E. Gabrielli, S. Khalil, C. Muñoz and E. Torrente-Lujan, Phys. Rev. D63 (2001) 025008 .

[25] D. Bailin, G.V. Kraniotis and A. Love, Phys. Lett. B491 (2000) 161.

[26] R. Bernabei et al., Phys. Lett. B480 (2000) 23.

[27] R. Abusaidi et al., Phys. Rev. Lett. 84 (2000) 5699.

[28] A. Bottino, F. Donato, N. Fornengo and S. Scopel, Phys. Rev. D59 (1999) 095004; R. Arnowitt and P. Nath, Phys. Rev. D60 (1999) 044004; E. Accomando, R. Arnowitt, B. Dutta and Y. Santoso, Nucl. Phys. B585 (2000) 124.

[29] M.E. Gómez and J.D. Vergados, hep-ph/0012020. 
[30] L.E. Ibañez, hep-ph/9905349; I. Antoniadis, C. Bachas and E. Dudas, Nucl. Phys. B560 (1999) 93; N. Arkani-Hamed, S. Dimopoulos and J. March-Russell, hepth/9908146.

[31] For a review, see e.g.: A. Brignole, L.E. Ibanez and C. Muñoz, in the book 'Perspectives on Supersymmetry', World Scientific Publ. Co. (1998) 125, hepph/9707209, and references therein.

[32] U. Chattopadhyay and P. Nath, Phys. Rev. D53 (1996) 1648.

[33] T. Moroi, Phys. Rev. D53 (1996) 6565; 56, 4424(E) (1997).

[34] M. Carena, G.F. Giudice, and C.E.M. Wagner, Phys. Lett. B390 (1997) 234; U. Chattopadhyay, D.K. Ghosh, and S. Roy, Phys. Rev. D62, 115001 (2000); K.T. Mahanthappa and S. Oh, Phys. Rev. D62 (2000) 015012.

[35] E. Gabrielli and U. Sarid, Phys. Rev. Lett. 79 (1997) 4752;

[36] J.L. Lopez, D.V. Nanopoulos and X. Wang, Phys. Rev. D49 (1991) 366.

[37] Z. Kakushadze, Phys. Lett. B434 (1998) 269; J. Lykken, E. Poppitz, S. Trivedi, Nucl. Phys. B543 (1999) 105.

[38] G. Aldazabal, L.E. Ibáñez, F. Quevedo, hep-ph/0001083; M. Cvetic, M. Plümacher, J. Wang, JHEP 0004 (2000) 004; G. Aldazabal, L.E. Ibáñez, F. Quevedo and A.M. Uranga, JHEP 0008 (2000) 002; D. Bailin, G.V. Kraniotis and A. Love, hep-th/0011289; S.F. King and D.A.J. Rayner, hep-ph/0012076.

[39] A. Brignole, L.E. Ibáñez and C. Muñoz, Nucl. Phys. B422 (1994) 125, B436 (1995) 747 (E); A. Brignole, L.E. Ibáñez, C. Muñoz and C. Scheich, Z. Phys. C74 (1997) 157.

[40] S. Abel, S. Khalil and O. Lebedev, hep-ph/0103031; hep-ph/0103320.

[41] S. Baek, P. Ko and H.S. Lee, hep-ph/0103218. 